\title{
Mídias sociais e suicídio
}

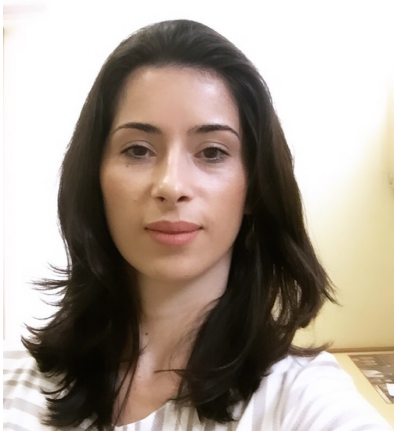

As mídias sociais, por meio de sites e aplicativos, permitem que usuários criem e compartilhem conteúdos ou participem de redes sociais. Elas são amplamente difundidas e modificaram intensamente as formas de interação entre os indivíduos ${ }^{(1)}$. As mídias sociais estão entre os múltiplos fatores de risco ou proteção para a saúde mental e o comportamento suicida, embora não possam isoladamente explicar esses fenômenos multifatoriais e complexos ${ }^{(2)}$.

$\mathrm{Na}$ internet, o tema suicídio pode ser um "ponto de encontro" entre indivíduos vulneráveis, pois estudos revelaram que jovens com problemas de saúde mental são usuários mais pesados de mídias sociais ${ }^{(3)}$ e pessoas que postam conteúdos sobre suicídio preferem usar blogs e fóruns on-line para se expressar e tendem a ser mais jovens, ter mais ideação suicida e afetividade negativa em comparação com pessoas sem postagens sobre suicídio(4).

A forma de abordar o suicídio em mídias precisa ser cuidadosa para evitar o efeito Werther ou contágio, que é um fenômeno de aparente disseminação de influências promotoras do comportamento suicida(5-6). Todavia, nas mídias sociais, conteúdos pró-suicidas podem ser produzidos anonimamente e são facilmente acessados por pessoas vulneráveis. Entre esses conteúdos estão os pactos de suicídio, jogos suicidas, manuais sobre métodos relacionados ao suicídio, bem como conteúdos que valorizam, romantizam, encorajam, condenam o suicídio ou dificultam a compreensão desse comportamento ${ }^{(7-8)}$.

O uso de mídias sociais pode ser prejudicial especialmente quando é intenso, associado a cyberbullying, exposição excessiva de intimidade, expectativas não realistas, percepção aumentada da felicidade e sucesso alheio, procrastinação, falta de crítica, reflexão e de atitudes empáticas e responsáveis ${ }^{(1,4,9-12)}$.

A intensidade e priorização da vida virtual também está associada a diversos prejuízos como sentimentos de inadequação, insatisfação com a imagem corporal, sintomas depressivos e ansiosos, pior qualidade do sono e a necessidade de engajamento excessivo nas mídias pelo medo de perder oque nelas ocorre ${ }^{(1,4,9-12)}$. Considerando esses potenciais efeitos negativos das mídias sociais, é importante o desenvolvimento de pesquisas e intervenções relacionadas a ações educativas e de apoio para jovens, pais e educadores.

É preciso considerar também as potencialidades das mídias para a promoção da saúde mental e prevenção do suicídio. A incorporação de estratégias virtuais criativas e atrativas aos cuidados convencionais pode ser acessível, facilitar a adesão de jovens, as trocas de experiências, expressão e rastreamento de emoções e necessidades, catalisação de apoio e pertença, maior difusão de informações, conhecimento sobre repertórios de enfrentamento e melhores resultados aos cuidados em saúde(7-8). Existem diferentes recursos com tal finalidade, tais como: grupos de apoio, aplicativos, serious games, sites. Além disso, há situações em que o anonimato pode facilitar a busca inicial por apoio.

É importante investir em pesquisas, inovações tecnológicas e ações educativas e de cuidado criativas, acessíveis, eficientes e adaptadas para públicos diversos para que as mídias sociais sejam utilizadas de forma positiva, segura em prol da prevenção do suicídio e a promoção da saúde mental. 


\section{Referências}

1. Royal Society for Public Health. Social media and young people's mental health and wellbeing. London: Royal Society for Public Health; 2017. 32 p. [cited 2018 Nov 23]. Available from: https://www.rsph.org.uk/uploads/assets/ uploaded/62be270a-a55f-4719-ad668c2ec7a74c2a.pdf

2. World Health Organization. Preventing suicide: a global imperative. WHO Press: Geneva, 2014 [cited 2018 Nov 23]. Available from: http://apps.who.int/iris/bitstream/handle/10665/131056/9789241564779_eng.pdf;jsessionid=588169C 76F2329F73230A31BF4DC51AE?sequence=1

3. Sampasa-Kanyinga H, Lewis RF. Frequent Use of Social Networking Sites Is Associated with Poor Psychological Functioning Among Children and Adolescents. Cyberpsychology, Behav Soc Netw. 2015; Jul;18(7):380-5. doi: 10.1089/ cyber.2015.0055

4. Cheng Q, Kwok CL, Zhu T, Guan L, Yip PSF. Suicide communication on social media and its psychological mechanisms: An examination of chinese microblog users. Int J Environ Res Public Health. 2015; Sep;12(9):11506-27. doi: 10.3390/ijerph120911506.

5. Cheng Q, Li H, Silenzio V, Caine ED. Suicide contagion: A systematic review of definitions and research utility. PLoS One. 2014; Sep; 9(9): e108724. doi.org/10.1371/journal.pone.0108724

6. Robinson J, Cox G, Bailey E, Hetrick S, Rodrigues M, Fisher S, et al. Social media and suicide prevention: A systematic review. Early Interv Psychiatry. 2016; Apr;10(2):103-21. doi: 10.1111/eip.12229. Epub 2015 Feb 19.

7. Vedana KGG, Pereira CCM, Di Donato G, Vanzela AS. 13 Reasons Why": social blog posts about the book and series related to suicidal behavior among young individuals. Enferm Rev. 2018;21(1):2-10. [cited 2018 Nov 23]. Available from: http://periodicos.pucminas.br/index.php/enfermagemrevista/article/view/17891/13345

8. Vedana KGG, Silva AF, Pereira CCM, Silva GL. "Blue Whale": blog posts about the suicide game. Rev Enferm Contemp. 2018;7(1):27. [cited 2018 Nov 23]. Available from: https://www5.bahiana.edu.br/index.php/enfermagem/ article/view/1736

9. Braithwaite SR, Giraud-Carrier C, West J, Barnes MD, Hanson CL. Validating Machine Learning Algorithms for Twitter Data Against Established Measures of Suicidality. JMIR Ment Heal [Internet]. 2016 May; 16;3(2):e21. doi: $10.2196 /$ mental.4822.

10. Daine K, Hawton K, Singaravelu V, Stewart A, Simkin S, Montgomery P. The power of the web: a systematic review of studies of the influence of the internet on self-harm and suicide in young people. PLoS One. 2013;Oct; 8(10): e77555. doi.org/10.1371/journal.pone.0077555

11. Li TMH, Chau M, Yip PSF, Wong PWC. Temporal and Computerized Psycholinguistic Analysis of the Blog of a Chinese Adolescent Suicide. Crisis. 2014 May; 35(3):168-75. doi: 10.1027/0227-5910/a000248.

12. Mars B, Heron J, Biddle L, Donovan JL, Holley R, Piper M, et al. Exposure to, and searching for, information about suicide and self-harm on the Internet: Prevalence and predictors in a population based cohort of young adults. J Affect Disord. Oct 1;185:239-45. doi: 10.1016/j.jad.2015.06.001. Epub 2015 Jun 12.

Kelly Graziani Giacchero Vedana

Professor Doutor, Escola de Enfermagem de Ribeirão Preto, Universidade de São Paulo, Centro Colaborador da OPAS/OMS para o Desenvolvimento da Pesquisa em Enfermagem, Ribeirão Preto, SP, Brasil.

E-mail: kellygiacchero@eerp.usp.br

(iD) https://orcid.org/0000-0001-7363-2429 